\title{
British Museum, Research Laboratory
}

\begin{tabular}{|c|c|c|c|c|c|}
\hline $\begin{array}{l}\text { Laboratory } \\
\text { number }\end{array}$ & $\begin{array}{l}\text { Published } \\
\text { reference }\end{array}$ & $\begin{array}{l}\text { Original date or } \\
\text { other value }\end{array}$ & $\begin{array}{l}\text { Corrected date } \\
\text { or other value }\end{array}$ & $\partial \mathrm{C}^{14}, \%$ & $\begin{array}{c}\text { A.D./ } \\
\text { B.C. date }\end{array}$ \\
\hline BM- $15^{1}$ & $54: 82$ & $2080 \pm 150$ & & & 130 B.C. \\
\hline $\begin{array}{r}-16 \\
-16\end{array}$ & $54: 82$ & $1333 \pm 150$ & & & A.D. 617 \\
\hline-17 & $54: 85$ & $1570 \pm 150$ & see $B M-47$ & & A.D. 380 \\
\hline$-18^{2}$ & $54: 83$ & $2803 \pm 150$ & & & 853 B.C. \\
\hline$-19^{3}$ & $54: 83$ & $11,333 \pm 200$ & & & 9,383 B.C. \\
\hline-21 & $54: 85$ & $3580 \pm 150$ & & & 1630 B.C. \\
\hline$-22^{4}$ & $54: 83$ & $3530 \pm 150$ & & & 1580 B.C. \\
\hline-27 & $54: 85$ & $4100 \pm 150$ & & & 2150 B.C. \\
\hline-29 & $54: 85$ & $3656 \pm 150$ & & & 1706 B.C. \\
\hline-30 & $54: 83$ & $18,200 \pm 500$ & & & 16,250 B.C. \\
\hline $\begin{array}{l}-30 \\
-31\end{array}$ & $\begin{array}{l}54: 83 \\
56: 39\end{array}$ & $\begin{array}{r}10,200 \pm 150 \\
1020 \pm 150\end{array}$ & & & A.D. 930 \\
\hline .34 & $54: 84$ & $4490 \pm 150$ & & & 2540 B.C. \\
\hline-37 & $54: 84$ & $920 \pm 150$ & & & A.D. 1030 \\
\hline-39 & $54: 84$ & $>25,000$ & & & $>23,000$ B.C \\
\hline-40 & $54: 85$ & $5660 \pm 150$ & & & 3710 B.C. \\
\hline $\begin{array}{l}-40 \\
-41\end{array}$ & $\begin{array}{l}54: 03 \\
55: 29\end{array}$ & $1810 \pm 150$ & & & A.D. 140 \\
\hline-42 & $56: 39$ & $870 \pm 150$ & & & A.D. 1080 \\
\hline-43 & $55: 29$ & $3450 \pm 150$ & & & 1500 B.C. \\
\hline-46 & $55: 27$ & $3670 \pm 150$ & & & 1720 B.C. \\
\hline-47 & $55: 27$ & $2410 \pm 150$ & & & 460 B.C. \\
\hline-48 & $55: 29$ & $7550 \pm 180$ & & & 5600 B.C. \\
\hline-49 & $55: 28$ & $4680 \pm 150$ & & & 2730 B.C. \\
\hline-52 & $55: 26$ & $100 \pm 150$ & & & recent \\
\hline-54 & $55: 29$ & $4120 \pm 150$ & & & 2170 B.C. \\
\hline-56 & $55: 27$ & $1500 \pm 150$ & & & A.D. 450 \\
\hline-57 & $55: 27$ & $4200 \pm 150$ & & & 2250 B.C. \\
\hline-58 & $55: 28$ & $2700 \pm 150$ & & & 750 B.C. \\
\hline$-59^{5}$ & $55: 30$ & $2400 \pm 150$ & & & 450 B.C. \\
\hline-62 & $55: 28$ & $3750 \pm 150$ & & & 1800 B.C. \\
\hline-63 & $55: 28$ & $2400 \pm 150$ & & & 450 B.C. \\
\hline-64 & $56: 43$ & $3920 \pm 150$ & & & 1970 B.C. \\
\hline .65 & $55: 29$ & $8100 \pm 180$ & & & 6150 B.C. \\
\hline $\begin{array}{l}-0.3 \\
-67\end{array}$ & $\begin{array}{l}50: 29 \\
55: 27\end{array}$ & $1120 \pm 150$ & & & A.D. 830 \\
\hline-68 & $56: 40$ & $3530 \pm 150$ & & & 1580 B.C. \\
\hline .70 & $56: 43$ & $4030 \pm 150$ & & & 2080 B.C. \\
\hline-73 & $56: 42$ & $4910+150$ & & & 2960 B.C. \\
\hline .74 & $56: 42$ & $4530 \pm-150$ & & & 2580 B.C. \\
\hline .75 & $56: 42$ & $3500 \pm 150$ & & & 1550 B.C. \\
\hline .76 & $56: 43$ & $3990 \pm 150$ & & & 2040 B.C. \\
\hline .78 & $56: 43$ & $4260 \pm 150$ & & & 2310 B.C. \\
\hline .79 & $56: 40$ & $2130 \pm 150$ & & & 180 B.C. \\
\hline .80 & $56: 41,42$ & $28700 \pm 180$ & & & 6750 B.C. \\
\hline-81 & $56: 42$ & $8680 \pm 180$ & see $B M-80$ & & 6730 B.C. \\
\hline-82 & $56: 40$ & $3950 \pm 150$ & & & 2000 B.C. \\
\hline-83 & $56: 42$ & $8110 \pm 180$ & see $B M-80$ & & 6160 B.C. \\
\hline-85 & $56: 43$ & $2400 \pm 150$ & & & 450 B.C. \\
\hline-86 & $56: 41$ & $3310 \pm 150$ & & & 1360 B.C. \\
\hline-87 & $56: 41$ & $4270 \pm 150$ & & & 2320 B.C. \\
\hline-88 & $56: 41$ & $4050 \pm 150$ & & & 2100 B.C. \\
\hline-89 & $56: 40$ & $6450 \pm 150$ & & & 4500 B.C. \\
\hline-90 & $56: 42$ & $8100 \pm 180$ & see $B M-80$ & & 6150 B.C. \\
\hline-92 & $56: 42$ & $2240 \pm 150$ & & & 290 B.C. \\
\hline-93 & $109: 106$ & $3870 \pm 150$ & & & 1920 B.C. \\
\hline .95 & $56: 44$ & $680 \pm 150$ & & & A.D. 1270 \\
\hline-97 & 109:106 & $4290 \pm 150$ & & & 2340 B.C. \\
\hline .99 & 109:106 & $3980 \pm 150$ & & & 2030 B.C. \\
\hline-100 & 109:107 & $4660 \pm 150$ & & & 2710 B.C. \\
\hline-101 & 109:107 & $4485 \pm 150$ & & & 2535 B.C. \\
\hline-103 & $109: 106$ & $3700 \pm 150$ & & & 1750 B.C. \\
\hline
\end{tabular}




\begin{tabular}{|c|c|c|c|c|c|}
\hline $\begin{array}{l}\text { Laboratory } \\
\text { number }\end{array}$ & $\begin{array}{l}\text { Published } \\
\text { reference }\end{array}$ & $\begin{array}{l}\text { Original date or } \\
\text { other value }\end{array}$ & $\begin{array}{l}\text { Corrected date } \\
\text { or other value }\end{array}$ & $\partial \mathrm{C}^{14}, \%$ & $\begin{array}{c}\text { A.D./ } \\
\text { B.C. date }\end{array}$ \\
\hline .105 & 109:107 & $10,250 \pm 200$ & & & 8300 B.C. \\
\hline -106 & 109:107 & $10,300 \pm 200$ & & & 8350 B.C. \\
\hline . 109 & 109:106 & $3290 \pm 150$ & & & 1340 B.C. \\
\hline-110 & 109:107 & $10,180 \pm 200$ & & & 8230 B.C. \\
\hline-113 & 109:105 & $4660 \pm 150$ & & & 2710 B.C \\
\hline .115 & $109: 107$ & $9170 \pm 200$ & & & 7220 B.C. \\
\hline-122 & 109:105 & $2960 \pm 150$ & & & 1010 B.C. \\
\hline-123 & $109: 105$ & $2890 \pm 150$ & & & 940 B.C. \\
\hline-124 & 109:104 & $8050 \pm 180$ & & & 6100 B.C. \\
\hline-125 & 109:108 & $7770 \pm 180$ & & & 5820 B.C. \\
\hline-126 & $109: 104$ & $7000 \pm 180$ & & & 5050 B.C. \\
\hline-127 & 109:108 & $8700 \pm 180$ & & & 6750 B.C. \\
\hline-128 & 109:104 & $850 \pm 150$ & & & A.D. 1100 \\
\hline-129 & $109: 105$ & $4440 \pm 150$ & & & 2490 B.C. \\
\hline-130 & 109:106 & $5100 \pm 150$ & & & 3150 B.C \\
\hline-133 & $109: 105$ & $3800 \pm 150$ & & & $1850 \mathrm{~B}$ \\
\hline
\end{tabular}

${ }^{1}$ St-103A, Q-112, U-68.

${ }^{2} \mathrm{C}-159$.

${ }^{3}$ K-101, U-20, U-75, St-18.

${ }^{4} \mathrm{C}-81$.

${ }^{5}$ D-70.

\section{University of Chicago, Institute for Nuclear Studies}

\begin{tabular}{|c|c|c|c|c|c|}
\hline $\begin{array}{l}\text { Laboratory } \\
\text { number }\end{array}$ & $\begin{array}{l}\text { Published } \\
\text { reference }\end{array}$ & $\begin{array}{l}\text { Original date } \\
\text { or other value }\end{array}$ & $\begin{array}{c}\text { Corrected date } \\
\text { or other value }\end{array}$ & $\partial \mathrm{C}^{14}, \%$ & $\begin{array}{c}\text { A.D./ } \\
\text { B.C.date }\end{array}$ \\
\hline C-1 & a95: $77^{1}$ & $3979 \pm 350 \mathrm{~s}$ & & & 2029 B.C. \\
\hline-12 & a95:77 & $4802 \pm 210^{\mathrm{s}}$ & & & 2852 B.C. \\
\hline-36 & a95:90 & $876 \pm 250 \mathrm{~s}$ & & & A.D. 1074 \\
\hline-37 & a95:90 & $1800 \pm 500 \mathrm{~s}$ & & & A.D. 150 \\
\hline-38 & a95:90 & $5159 \pm 350 \mathrm{~s}$ & & & 3209 B.C. \\
\hline-39 & a95:90 & $8323 \pm 400^{\mathrm{s}}$ & & & 6373 B.C. \\
\hline-62 & a95:77 & $2190 \pm 450^{\mathrm{s}}$ & & & 240 B.C. \\
\hline-65 & a95:106 & $5356 \pm 350^{\mathrm{s}}$ & & & 3306 B.C. \\
\hline-72 & a95:83 & $2531 \pm 150^{\mathrm{s}}$ & & & 581 B.C. \\
\hline-75 & a95:133 & $2665 \pm 200 \mathrm{~s}$ & & & 715 B.C. \\
\hline-81 & a95:77 & $3621 \pm 180^{\mathrm{s}}$ & & & 1671 B.C. \\
\hline-101 & a95:127 & ITI + $14011 \%$ & see C-112 & & A.D. 417 \\
\hline-102 & a95:127 & & see C-112 & & A.D. 417 \\
\hline-103 & a95:134 & $1042 \pm 80^{\mathrm{s}}$ & & & A.D. 908 \\
\hline-105 & a95:105 & $>20,000$ & & & \\
\hline$-108 a$ & a95:107 & $8274 \pm 500^{\mathrm{s}}$ & & & 6324 B.C. \\
\hline-112 & a95:127 & $1533 \pm 150^{\mathrm{s}}$ & & & A.D. 417 \\
\hline-113 & a95:79 & $6707 \pm 320^{\mathrm{s}}$ & & & 4757 B.C. \\
\hline-115 & a95:79 & $3212 \pm 250^{\mathrm{s}}$ & & & 1262 B.C. \\
\hline-116 & a95:94 & $5149 \pm 300^{\mathrm{s}}$ & & & 3199 B.C. \\
\hline-119 & a95:90 & $2141 \pm 250^{\mathrm{s}}$ & & & 191 B.C. \\
\hline-120 & a95:90 & $5305 \pm 250^{\mathrm{s}}$ & & & 3355 B.C. \\
\hline-121 & a95:90 & $\mathrm{d}$ & & & \\
\hline-122 & a95:90 & $6668 \pm 250^{\mathrm{s}}$ & & & 4718 B.C. \\
\hline-126 & a95:94 & $1168 \pm 150^{\mathrm{s}}$ & & & A.D. 782 \\
\hline-136 & a95:94 & $1951 \pm 200^{\mathrm{s}}$ & & & 1 B.C. \\
\hline-137 & a95:94 & $2285 \pm 210^{\mathrm{s}}$ & & & 335 B.C. \\
\hline-139 & a95:95 & $2044 \pm 250^{\mathrm{s}}$ & & & 94 B.C. \\
\hline-143 & a95:106 & $1158 \pm 250^{\mathrm{s}}$ & & & A.D. 782 \\
\hline-150 & a95:106 & $633 \pm 150^{\mathrm{s}}$ & & & A.D. 1317 \\
\hline .151 & a95:106 & $1233 \pm 250^{\mathrm{s}}$ & & & A.D. 717 \\
\hline-152 & a95:95 & $2336 \pm 250^{\mathrm{s}}$ & & & 386 B.C. \\
\hline-153 & b95:108 & $1553 \pm 175^{\mathrm{s}}$ & & & A.D. 397 \\
\hline-154 & a95:106 & $1276 \pm 150 \mathrm{~s}$ & & & A.D. 674 \\
\hline-159 & a95:134 & $2710 \pm 130 \mathrm{~s}$ & & & 760 B.C. \\
\hline
\end{tabular}

